\title{
Implementação de Laboratórios Virtuais no metaverso OpenSim
}

\author{
Bárbara Ávila ${ }^{1}$, Érico M. H. Amaral ${ }^{1,2}$, Liane Tarouco ${ }^{1}$ \\ ${ }^{1}$ Programa de Pós-graduação em Informática na Educação - Universidade Federal do \\ Rio Grande do Sul (UFRGS) \\ ${ }^{2}$ Universidade Federal dos Pampas (Unipampa) \\ barbaragorzizaavila@gmail.com, ericohoffamaral@gmail.com, \\ liane@penta.ufrgs.br
}

\section{Resumo}

Este artigo discute a viabilização de laboratórios virtuais de aprendizagem dentro do metaverso OpenSim. Neste contexto, são apresentadas investigações técnicas inerentes ao processo, onde se destacam os suportes oferecidos pelos diferentes viewers, a importação de objetos para dentro do metaverso e a adição de scripts, com os quais visase a alcançar maior nível de interatividade. Adicionalmente, são apresentados os processos de implementação de um laboratório de Geometria e os resultados obtidos até o presente momento.

\section{Palavras-chave}

Ambientes Imersivos, Metaversos, Laboratórios Virtuais, VEGA.

\section{Implementing Virtual Laboratories in OpenSim metaverse}

\begin{abstract}
This paper discusses the viability of virtual learning labs within the OpenSim metaverse. In this context, we present investigations techniques inherent to the process, where we highlight the supports offered by different viewers, importing objects into the metaverse and adding scripts, with which it aims to achieve a higher level of interactivity. Addititionally, we present the implementation processes of a laboratory Geometry and results to date.
\end{abstract}

\section{Keywords}

Immersive Environments, Metaverses, Virtual Laboratories, VEGA.

\section{Introdução}

Ambientes imersivos, cada vez mais explorados no campo das tecnologias para entretenimento, vêm também conquistando espaço no âmbito educacional. Situações de ensino e aprendizagem, calcadas na experiência prática, encontram nos metaversos espaços muito propícios para o seu desenvolvimento devido ao alto nível de interatividade oferecido pelos mundos virtuais.

Neste contexto, a proposta de viabilização de laboratórios de aprendizagem através destes mundos vem se apresentando como algo promissor e digno de pesquisa para muitos membros da comunidade acadêmica.

Este é o intuito do presente trabalho, que visa construir um laboratório virtual de Geometria, a ser oferecido como um espaço complementar às atividades escolares. Para concretizar o desenvolvimento deste laboratório, uma série de investigações técnicas vem 
sendo conduzidas, e serão relatadas ao longo deste artigo, que está organizado da seguinte maneira: na seção 2 discutem-se as potencialidades e limitações dos mundos virtuais, no que tange à implementação de laboratórios de aprendizagem. Na seção 3 é apresentada a metodologia utilizada para as pesquisas conduzidas até o presente momento. A seção 4 traz o processo de implementação de um laboratório, ainda em desenvolvimento. A seção 5 apresenta os resultados já alcançados e finaliza-se com a seção 6, onde são discutidas as considerações finais do trabalho.

\section{Metaversos, suas Características e Limitações}

Nesta seção serão abordadas as principais características dos Mundos Virtuais, com uma visão teoricamente fundamentada, sobre seus aspectos de imersividade, considerações sobre a aprendizagem nestes ambientes e, por fim, uma descrição técnica de suas funcionalidades e limitações.

\subsection{Laboratórios Virtuais de Aprendizagem em ambientes imersivos}

Atividades baseadas em computador, quando elaboradas com propósitos claros, tendem a ensejar uma interação ativa do estudante com o objeto de conhecimento, ao mesmo tempo em que lhe proporcionam autonomia para determinar o ritmo de seu processo de aprendizagem. Este é um aspecto que ganha relevância em pesquisas como a de Fletcher (2001), na qual se propõe o uso de atividades baseadas em computador com vistas a oferecer tutorias individualizadas, cuja eficiência já fora destacada por Bloom (1984).

Com a disseminação da internet, a Comunicação Mediada por Computador (CMC) abriu caminho para novas estratégias pedagógicas pautadas em práticas colaborativas, cuja importância no processo de aprendizagem já foi fortemente destacada por teóricos como (Vygotsky, 2008; Jonassen, et al., 1999)

Características como a possibilidade de colaboração, de participação ativa do estudante, incentivo à investigação e à resolução de problemas costumam ser inerentes aos laboratórios de aprendizagem que, com os avanços tecnológicos, vêm sendo oferecidos através dos ambientes virtuais.

Autores como (Callaghan et al., 2008; Ramos-Paja, Scarpetta, Martínez-Salamero, 2010) apontam que diversos fatores contribuem para que os estudantes nem sempre disponham de um acesso direto a laboratórios para a realização de seus experimentos. Entretanto, tais práticas são imprescindíveis para profissões que deverão requerer a manipulação de instrumentos, como no caso das engenharias ou da própria medicina, ou mesmo para a formulação de conceitos abstratos que podem ser compreendidos a partir da observação de objetos ou fenômenos, como ocorre na aprendizagem da Física ou da Matemática. Desse modo, dispor de um acesso remoto a laboratórios de aprendizagem torna-se uma opção eficiente para aqueles que não têm condições de se deslocar até a instituição. Tais laboratórios, viabilizados através da web, podem ser do tipo Remotos (LVRs) ou Virtuais (LVAs) e caracterizam-se por não requererem a presença do estudante na instituição de ensino.

Os Laboratórios Remotos oferecem uma interface que permite a manipulação a distância de instrumentos disponíveis no laboratório real. O ingresso nestes ambientes costuma se dar via autenticação do aluno, que deve aguardar pela liberação de acesso aos instrumentos e experimentos desejados. Como exemplo para este tipo de laboratório Callaghan et al. (2007) citam o projeto Diesel, que evoluiu de um ambiente onde os alunos tinham acesso isoladamente a um espaço colaborativo onde todos os estudantes passaram a interagir em uma mesma experiência, com o apoio de recursos de áudio e vídeo. 
Segundo os autores, o incentivo à comunicação e à colaboração promoveram no grupo uma maior sensação de proximidade, enriquecendo o seu desempenho na condução dos experimentos.

Ainda como exemplo de Laboratórios Remotos, pode-se citar o autorama do Weblab, desenvolvido por Sievers et al. (2011) para o estudo do Movimento Uniformemente Variado, a nível de Ensino Médio.

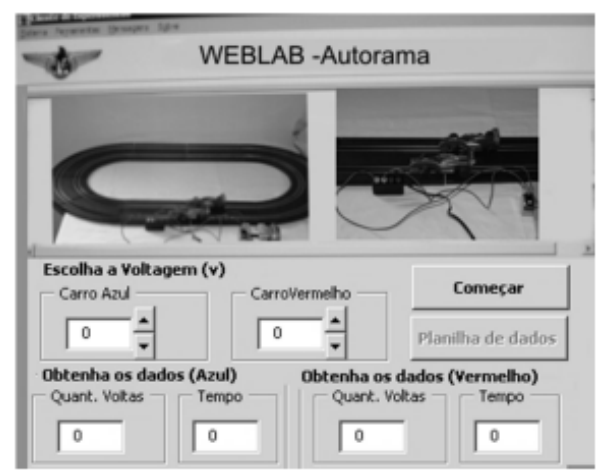

Figura 1 - Autorama desenvolvido no Weblab por Sievers Jr. et al. (2011)

Os Laboratórios Virtuais de Aprendizagem, por sua vez, consistem em representações virtuais que reproduzem o ambiente de um laboratório real. Tratam-se de aplicações totalmente baseadas em simulações, nas quais se dispõe somente de representações computacionais da realidade. Por não haver limitações referentes ao número de instrumentos ou experimentos disponíveis nesta modalidade, não há necessariamente uma obrigatoriedade de espera para a participação em um experimento, não se fazendo necessária a reserva de horário para uso do laboratório.

Exemplos deste tipo de aplicação são o Laboratório Virtual da Unijuí ${ }^{1}$ e o E2D ${ }^{2}$, sendo que ambos consistem em páginas web com exercícios interativos e propostas de investigação no âmbito da Matemática.

Uma nova proposta para a construção de espaços educacionais colaborativos surgiu com os Ambientes Imersivos, que consistem em sistemas gráficos 3D com alta capacidade de renderização, e que promovem uma interação imersiva do usuário com o sistema. Exemplos desta imersão podem ser encontrados em jogos eletrônicos como Wii, Xbox e PlayStation, nos quais usuários interagem com o ambiente através de diferentes dispositivos capazes de capturar seus movimentos e reproduzi-los no mundo virtual. Ainda no âmbito dos jogos eletrônicos, os mundos virtuais (ou Metaversos) têm se apresentado como uma opção capaz de engajar o usuário em experiências também bastante realísticas. Visando-se explorar tal capacidade de manter a atenção e o envolvimento dos usuários, vêm se consolidando na academia pesquisas sobre o uso dos Metaversos nos mais diversos contextos educacionais.

Os Metaversos (meta universos) (Schmitt e Tarouco, 2008) são mundos virtuais tridimensionais, nos quais os usuários transitam, interagem e vivenciam experiências, representados por seus avatares. Os avatares podem assumir a forma humana, ou adotar outros seres como animais, alienígenas, e tantas outras opções. Além das opções de avatares oferecidas pelos mundos virtuais, estes ainda podem ser personalizados através de ferramentas que permitem a alteração de sua aparência, como características físicas,

\footnotetext{
${ }^{1}$ http://www.projetos.unijui.edu.br/matematica/

${ }^{2}$ Ensino de Derivadas a Distância - ambiente elaborado pelos autores deste texto para apoio ao ensino de Cálculo. Disponível em http://www.scutum.com.br/e2d/index.html.
} 
vestimentas e acessórios adotados. Adicionada a estas opções de controle, quando atribuída autoria aos participantes, estes podem também construir ou importar objetos para dentro do mundo virtual.

Autores como Schaf, Suenoni e Pereira (2012) argumentam que este novo modelo de interface vem atraindo a formação de comunidades virtuais e, em decorrência disso, apresenta-se como um potencial recurso para o desenvolvimento de laboratórios de aprendizagem, visto que a concretização destes requer a existência de espaços colaborativos.

Tendo em vista a relevância da experiência concreta e da participação ativa do estudante em seu processo de aprendizagem (Kolb, 1986), é importante que se busque meios para viabilizar o seu contato com atividades que promovam um engajamento na investigação de situações-problema, baseadas em situações reais, onde o estudante conte com os benefícios de um ambiente colaborativo. Tal proposta remete às práticas pedagógicas comumente reproduzidas nos Laboratórios de Aprendizagem.

Nos Metaversos, espaços de aprendizagem com atividades laboratoriais podem ser consolidados com grande eficácia, devido às características realísticas dos mundos virtuais e suas amplas possibilidades colaborativas. Neste contexto, réplicas virtuais de laboratórios podem ser disponibilizadas, levando-se o mundo virtual a assumir o papel de uma sala de aula, onde os alunos realizam suas atividades, manipulando, construindo objetos e resolvendo problemas, conjuntamente com seus colegas, numa interação que pode ser estabelecida a distância, sem prejuízos no nível de envolvimento dos participantes.

Tomando por base esta perspectiva, a presente pesquisa visa a construção de laboratórios virtuais dentro do metaverso OpenSim. Vislumbra-se permitir não somente a interação do usuário com os elementos disponibilizados no mundo virtual, mas também que este possa criar objetos que o ajudem na resolução de problemas mal estruturados, explorados em pesquisas como de Wijekumar e Jonassen (2004).

Para tanto, vem se fazendo necessária uma ampla investigação acerca de metaversos existentes, com suas potencialidades e limitações. Esta experiência será relatada ao longo das seguintes seções.

\subsection{Diferentes viewers e suas limitações}

O acesso ao mundo virtual ocorre através de viewers, que oferecem uma interface entre o usuário e o metaverso. Os viewers possuem funcionalidades distintas, o que pode tornálos recomendáveis para determinadas atividades e, ao mesmo tempo, inadequados para outras.

Devido à sua compatibilidade com o Second Life (SL), o OpenSim (OS) pode utilizar os mesmos viewers, ainda que estes tenham sido construídos especificamente para o software proprietário (Leal, 2007). Dentre o conjunto de viewers disponíveis para o acesso aos metaversos SL e OS, estão: Imprudence, Firestorm, RealXtend, Hippo, Cool viewer, Second Life viewer, dentre outros.

Na pesquisa conduzida até o momento, quatro visualizadores foram amplamente inspecionados com o intuito de se definir qual seria mais recomendado para a construção de um laboratório virtual. Tendo em vista que a proposta deste laboratório consiste em um nível de autoria mais elevado para alunos e docentes que venham a interagir com este espaço, faz-se necessário oferecer soluções alternativas que não demandem alto conhecimento em programação na interação com o ambiente e ainda assim promovam o engajamento do usuário através de atividades mais interativas (Amaral et al., 2011). 
Para isso, conta-se com repositórios online que oferecem comercial ou gratuitamente objetos e animações que podem ser inseridos no mundo virtual e, em certo casos, associados aos avatares. Além disso, determinados softwares destinados à construção de objetos em três dimensões possibilitam a criação de objetos e animações pelo próprio usuário, que poderá importá-las para dentro do metaverso, como será discutido mais adiante.

As possibilidades de importação destes objetos varia muito de viewer para viewer, o que dificulta a escolha da ferramenta ideal para se adotar. Tendo em vista as peculiaridades apresentadas por cada uma das ferramentas disponíveis, a comunidade do OpenSim organizou uma tabela comparativa com funcionalidades e limitações comuns aos viewers mais conhecidos (Opensimulator, 2012). Esta tabela contém funções comuns a serem realizadas com os viewers no acesso aos mundos virtuais.

Nesta pesquisa, em particular, o ponto mais importante na comparação entre os viewers vem sendo as possibilidades de importação que oferecem, visto que busca-se contar não somente com as ferramentas internas do metaverso para a construção dos ambientes, mas também com ferramentas de autoria gratuitas e de acesso facilitado ao público usuário deste laboratório.

A partir dos viewers Imprudence e Hippo é possível, por exemplo, a importação de objetos disponibilizados gratuitamente em repositórios online no formato de arquivos .xml. Entretanto, não é possível a importação ou visualização de arquivos no formato “.dae”, provenientes de ferramentas para modelagem 3D como o SketchUp ou o Blender, restringindo as possibilidades de autoria dentro do laboratório às suas próprias ferramentas de edição ou a conhecimentos mais específicos de programação.

Esta questão de importação de arquivos no formato “.dae” já não é tão problemática em viewers como o Firestorm ou o RealXtend, que permitem a efetivação desta importação. Porém, ao contrário do Imprudence e do Hippo, já não se faz possível a importação dos arquivos .xml, disponíveis nos repositórios on line. A vantagem apresentada por estes viewers está no fato de que, apesar de não darem suporte à importação dos arquivos “.xml”, ainda assim possibilitam a sua visualização, oportunizando que um mundo seja composto com objetos de diferentes formatos que, embora importados através de diferentes viewers, são reconhecidos pelo Firestorm e pelo RealXtend.

Entretanto, ressalta-se que o processo de importação ainda não está totalmente livre de problemas. Objetos muito complexos costumam se desconfigurar no processo de importação e muitos não são devidamente reconhecidos pelo sistema. Por exemplo, sem interferência por via de programação, não é possível entrar em uma casa importada do SketchUp. Em função destes percalços, outros viewers, como o Singularity e o Second Life viewer ainda estão sob investigação no presente momento.

\subsection{Trabalhos Correlatos}

Conforme já mencionado, os mundos virtuais vêm despertando o interesse da comunidade acadêmica, sendo estes explorados nas mais diversas áreas do conhecimento.

Dentre as inúmeras pesquisas que envolvem o uso dos metaversos no contexto de ensino e aprendizagem, cabe destacar o Ecomuve, um mundo virtual desenvolvido por um grupo de pesquisadores de Harvard, destinado ao conhecimento sobre o funcionamento de ecossistemas (Dede, 2012). Neste projeto, foi construído um mundo virtual especificamente para a realização de estudos práticos sobre o meio ambiente. No mundo virtual, todos os elementos que compõem o ecossistema estão interligados, tal como no mundo real. Desse modo, abalos em determinados pontos repercutem em outros, 
alterando o funcionamento do sistema. Neste contexto, os estudantes deparam-se com elementos alterados e devem engajar-se em situações-problema, visando a identificar suas causas e possíveis soluções para o reestabelecimento do ecossistema. Trata-se de propostas educacionais que incentivam o envolvimento do estudante na resolução de situações que refletem acontecimentos da realidade, estabelecendo uma ligação com o contexto concreto, tal como Kolb (1986) sugere. Ao mesmo tempo, promove-se a participação colaborativa, visto que os estudantes compartilham o mundo e os problemas enfrentados dentro dele.

Mais voltado para o contexto de laboratórios de aprendizagem, baseados em réplica de instrumentos e experimentos presentes na instituição de ensino, tem-se o Laboratório Remoto, desenvolvido por Schaf, Suenoni e Pereira (2012). Este grupo de pesquisadores da Universidade Federal do Rio Grande do Sul desenvolveu um laboratório para o curso de Engenharia, utilizando-se do OpenSim como espaço virtual para a realização dos experimentos propostos. No mundo criado, foram disponibilizadas réplicas fidedignas de instrumentos disponíveis no laboratório real, na instituição de ensino. Ao interagir com tais instrumentos, no mundo virtual, os estudantes manipulavam a distância os instrumentos disponíveis na instituição. Ou seja, os estudantes tiveram a oportunidade de realizar a distância experiências reais no laboratório de sua instituição.

Além disso, Keskitalo, Pyykkö e Ruokamo (2011) vêm desenvolvendo uma proposta de modelo pedagógico denominada Global Virtual Education (GloVEd), utilizada para investigar a incidência de aprendizagem significativa em estudantes envolvidos em um projeto de Engenharia Civil, realizado no Second Life, cujo cerne consiste em promover o engajamento grupal na resolução de problemas, baseados no contexto real.

Tendo em vista pesquisas com este perfil e outras já mencionadas neste artigo, envolvendo de alguma forma a temática de laboratórios de aprendizagem e ambientes imersivos, este grupo de pesquisa passou a explorar possibilidades que efetivassem a construção de laboratórios dentro deste metaverso. Para tanto, vem sendo realizada uma intensa pesquisa com relação a ferramentas e métodos de implementação. Este tópico será abordado mais profundamente na seção seguinte, que trata sobre a metodologia do presente trabalho.

\section{Metodologia}

A metodologia proposta neste estudo prevê um levantamento teórico sobre os diferentes metaversos disponíveis, a fim de se definir uma infraestrutura de mundo virtual para o projeto. O mundo virtual a ser construído deverá constituir um Laboratório Virtual para o ensino de Geometria, utilizando-se deste tema como base para todas as demais etapas do estudo.

Com a escolha do metaverso, passa-se às ações empíricas desta pesquisa, com a avaliação e teste de importação e construção de objetos 3D, integração de softwares de autoria ao ambiente e, por último, implementação de scripts e agregação de funcionalidades interativas aos elementos inseridos no mundo virtual.

Ao final almeja-se alcançar um know how sobre as capacidades de um ambiente imersivo e suas potencialidades, além de fornecer uma descrição sobre a manipulação das tecnologias envolvidas. 


\section{Implementação do Mundo Virtual}

O estudo das diferentes ferramentas para construção de mundos virtuais permitiu elencar as tecnologias envolvidas neste tema, dando subsídios para a escolha da aplicação alinhada com a proposta desta pesquisa.

O OpenSim, por ser um software baseado no Second Life, gratuito e com uma comunidade forte, mostrou-se a escolha adequada neste momento, pois possui uma gama de soluções que podem ser utilizadas em conjunto com os metaversos criados com esta aplicação. Algumas delas se destacam como: diversos visualizadores, carga de objetos externos, construção de elementos a partir do próprio ambiente, utilização de diferentes linguagens de programação, facilidade na construção de scripts, entre outros. Estes detalhes serão descritos de forma clara nas seções 4.1 e 4.2.

\subsection{Importação de Objetos e Repositórios}

Conforme já mencionado, o OpenSim reconhece arquivos de diferentes formatos, sendo eles, porém, diretamente dependentes do suporte oferecido pelo viewer utilizado.

Ao trabalhar com viewers, como o Imprudence, pode-se contar com a importação de arquivos do tipo .xml, extensão com a qual são disponibilizados objetos em repositórios online, como o LindaKellie $^{3}$ e o OpenSim Creations ${ }^{4}$. O processo de importação é simples e os objetos são devidamente reconhecidos pelo metaverso, permitindo uma interação imediata do avatar com o objeto.

Apesar de ser animador o fato de se contar com repositórios que disponibilizam gratuitamente objetos a serem inseridos no mundo virtual, esta pesquisa prevê não somente um aluno usuário do espaço oferecido pelo laboratório, mas também autor, sendo-lhe propostas atividades que envolvem a elaboração de objetos. Neste caso, buscou-se transcender esta dependência dos produtores de conteúdo, através de ferramentas de autoria que facilitam o processo de elaboração e importação de objetos para dentro do OpenSim.

Para tanto, ferramentas como o SketchUp, o Blender e o Autocad podem facilitar a produção de conteúdo a partir da exportação de arquivos 3D para o formato .dae (Mesh Model), aceito no OpenSim por viewers como o Firestorm e o RealXtend. Embora o processo de importação não seja de grande complexidade, este grupo de pesquisa ainda encontra problemas com relação ao reconhecimento de alguns destes objetos, o que dificulta o planejamento de atividades que envolvem a interação com os mesmos.

\footnotetext{
${ }^{3}$ http://www.lindakellie.com/

$4 \underline{\text { http://opensim-creations.com/ }}$
} 


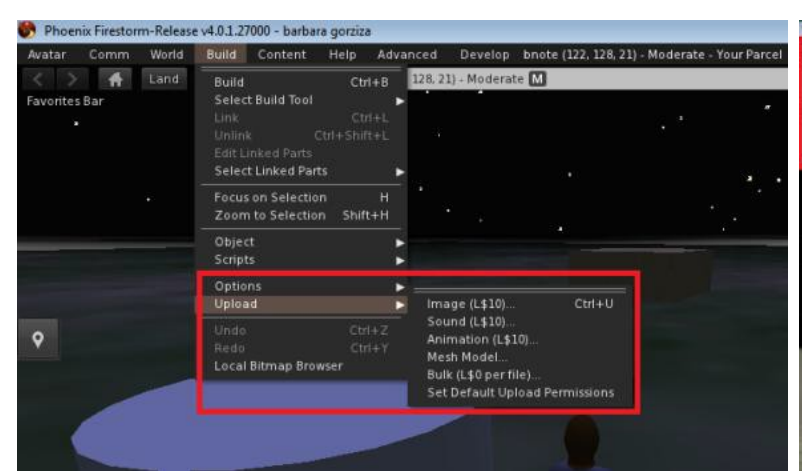

Figura 2. Processo de importação dos meshes (arquivos do tipo .dae) no viewer Firestorm

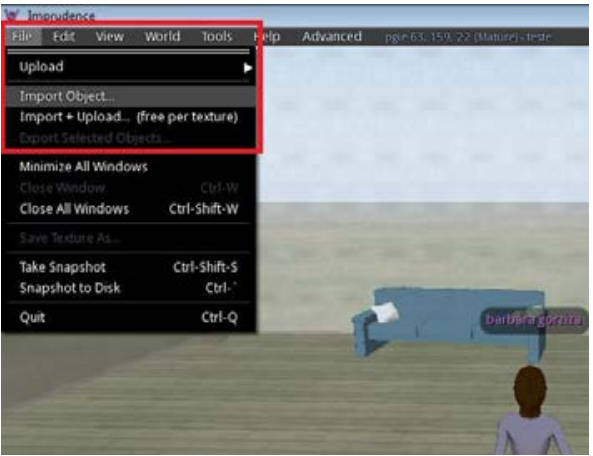

Figura 3. Processo de importação de arquivos do tipo .xml no viewer Imprudence

Atualmente, a atenção do grupo de pesquisa vem se detendo com ênfase nas questões referentes ao reconhecimento destes objetos, de modo a viabilizar o uso dos Meshes na composição do mundo virtual. Com o apoio destes recursos, vem sendo projetado um laboratório virtual de Geometria, o VEGA (Virtual Environment Guidance Acquaitance) que, em breve, deverá apresentar a sua primeira versão.

\subsection{Programação e Interatividade através de Scripts}

Uma característica do OpenSim é a capacidade de trabalhar com diferentes linguagens de programação como a própria Linden Script Language (LSL), linguagem desenvolvida inicialmente para o Second Life e herdada pelo OpenSim, além da OpenSim Scripting Language (OSSL), esta uma linguagem desenvolvida para o OS sendo uma extensão da LSL. Também destacam-se neste cenário as ferramentas de programação C\#, Java e Visual Basic, todas podendo ser ativadas de forma local, na versão stand alone, ou em um servidor OpenSim, na versão grid.

Em relação à sintaxe, as linguagens LSL e OSSL, possuem uma codificação muito próxima à linguagem $\mathrm{C}$, permitindo a compreensão dos programas de forma simples, garantindo desta forma a implementação de diferentes objetos e ações dentro do metaverso, segundo Morgado, Barbosa e Souza (2011). Embora todas as linguagens citadas possam ser utilizadas, a OSSL apresenta funcionalidades ímpares, as quais fazem parte de seu código fonte, citando-se os módulos Teleport Agent, que permite levar um avatar de um ponto a outro no mundo virtual, e a Set Dynamic Texture, URL que possibilita ser exibida, no próprio metaverso, uma imagem externa gerada dinamicamente.

Dentre as funcionalidades disponíveis no OS encontra-se a capacidade de executar scripts, geralmente com ações dentro do metaverso, a partir de interfaces específicas para isso. Esses scripts podem atingir qualquer objeto dentro do mundo virtual, independentemente de sua criação ou importação, funcionalidades estas que potencializam sua utilização como um espaço para simulação de ambientes 3D.

A programação dos scripts pode ser realizada de diferentes formas, porém existem aplicações que auxiliam nesta tarefa, tornando-a uma atividade menos complexa e até mesmo atraente. A seguir serão descritas duas ferramentas que corroboram para tal afirmação:

Uma das aplicações mais simples de serem utilizadas é o ScripTastic, um projeto desenvolvido pelo Greenbush Labs com intuito de fornecer uma interface básica, para 
implementação de scripts para o OpenSim. O ScripTastic utiliza uma interface sobre a plataforma javascript, que permite inserir ações em determinados objetos, realizando testes de forma rápida e dinâmica. Esta aplicação roda em um serviço web, porém a aplicação permite a carga dos códigos diretamente em HTML, javascript e CSS, para uma máquina local (Greenbush Labs, 2012). A Figura 04 apresenta a interface de acesso a aplicação, com os botões de controle de ação.

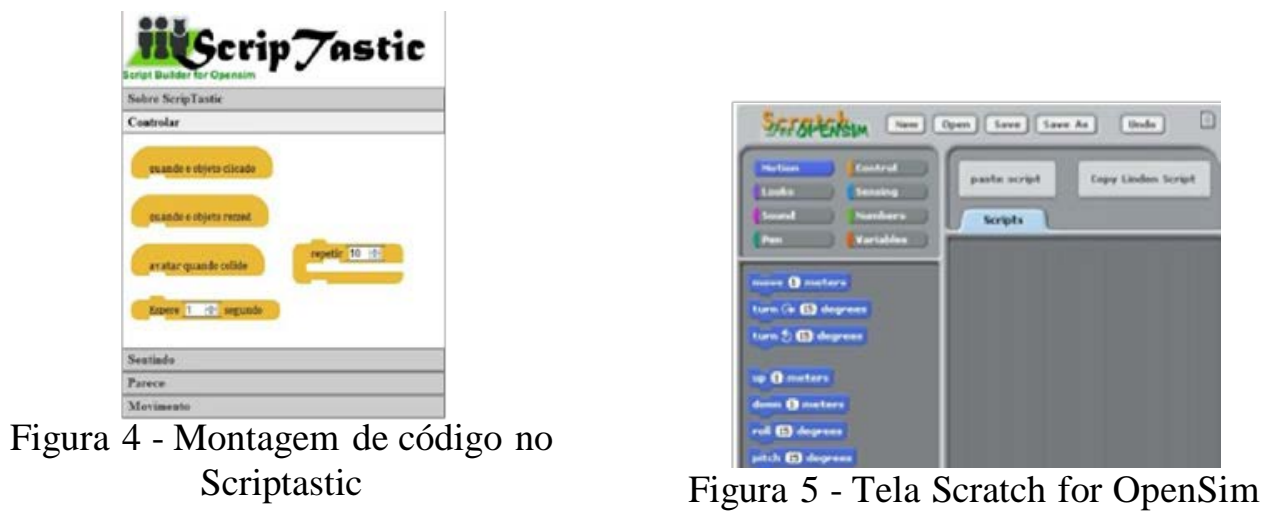

A segunda ferramenta descrita, Scratch for OpenSim, segue o mesmo princípio do ScripTastic. O Scratch for OpenSim é uma aplicação baseada em programação visual, onde o usuário precisa simplesmente definir as ações desejadas arrastando a aba com a função para que esta seja implementada. A partir disto, basta carregar para a área de memória o código construído e inseri-lo no metaverso. A Figura 05 demonstra a aplicação e seu conjunto de funcionalidades.

Cabe ressaltar que o Scratch for OpenSim é uma ferramenta amplamente utilizada por entusiastas fomentadores do uso de mundos virtuais, nas diferentes área do conhecimento, conforme descrito em http://www.frequency.com/video/scratch-4opensim-tutorial/28824534 e http://www.14l.co.uk/?p=551.

A efetiva implementação e utilização dos softwares descritos permite a construção de ambientes realmente imersivos, fornecendo um alto nível de interação aos usuários com um baixo nível de complexidade. Estes aspectos podem ser considerados de suma importância para a implementação de mundos atrativos e realmente interativos.

\section{Implementação e Resultados}

Para a implementação deste projeto e avaliação das potencialidades técnicas para o uso do OpenSim, partiu-se inicialmente de em um estudo teórico sobre o tema educação em ambientes imersivos, a fim de construir um contexto válido para as soluções e tecnologias a serem avaliadas. A partir do reconhecimento das funcionalidades deste metaverso foi possível partir para o desenvolvimento de um laboratório virtual para o ensino de Matemática, o qual vem permitindo o estudo e integração de um conjunto de tecnologias.

A primeira etapa constitui-se da avaliação dos métodos disponíveis para a instalação do OpenSim:

- O primeiro teste baseou-se na ativação do metaverso na versão StandAlone, a qual se caracteriza pelo simples download e execução da aplicação, com a posterior criação dos usuários do sistema e do mundo virtual propriamente dito.

- A segunda instalação avaliou o OpenSim na versão Grid ou servidora, onde um conjunto de passos mais complexos foi necessário, visto que esta opção se baseia na 
utilização de banco de dados para o armazenamento das informações do mundo virtual, neste caso utilizando o MySql como banco padrão, e da configuração de conexão com a rede para que os usuários possam acessar remotamente a aplicação.

Considerando que o próprio OS é um software desenvolvido com a tecnologia DotNet (Microsoft), o sistema base para o recebimento da aplicação deveria estar configurado para o correto funcionamento do metaverso, independente da plataforma nativa. Isto significa a necessidade do compilador DotNet, no mínimo na versão 3.5, para o Windows. No caso da instalação sob o Linux, é necessário a aplicação Mono rodando, para a emulação.

Com o mundo virtual ativo, partiu-se para a implementação do Laboratório Virtual denominado VEGA (Virtual Environment for Geometry Acquaintance) uma estrutura previamente projetada para servir como base de testes das tecnologias de mundos virtuais e atender requisitos de aprendizagem.

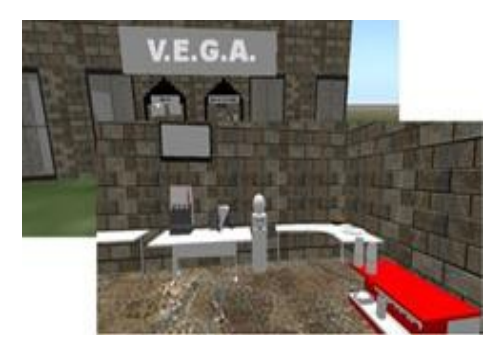

Figura 6 - Espaços disponíveis no laboratório VEGA

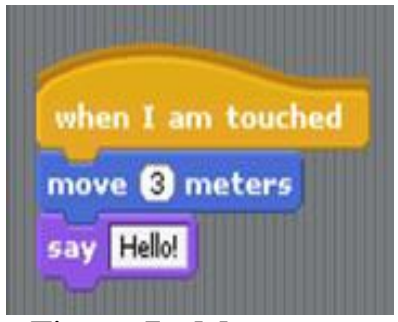

Figura 7 - Montagem de script no Scratch for OpenSim

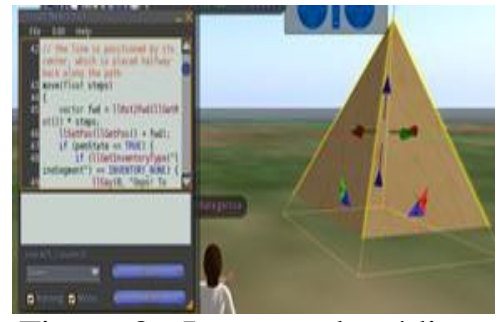

Figura 8 - Inserção de código no objeto

As estruturas 3D e objetos foram importadas a partir de arquivos .dae disponibilizados no SketchUp, os quais estão apresentados Figura 06. A integração de alguns softwares de autoria também foi avaliada a fim de verificar suas funcionalidades no contexto do metaverso, citando-se: o Adobe Flash, o Hot Potatoes e o Geogebra, os quais foram carregados por meio de uma plataforma web e validados de forma satisfatória.

Os testes relacionados à programação no metaverso estenderam-se à avaliação da linguagem de script OSSL, e suas funções nativas como os TeleportAgent(), que podem levar um avatar de um ponto a outro no mundo virtual, e os SetDynamicTextureURL(), que permitem a exibição no ambiente de imagens externas geradas de forma dinâmica. Sobre aspectos de interatividade, alguns scripts usando o Scratch for OpenSim foram implementados e testados. Tem-se as Figuras 07 e 08 como exemplos de tais testes, sendo a Figura 07 a construção das ações no Scratch e a Figura 08 apresentando a inserção do código em um determinado objeto.

A elaboração dos scripts para o OpenSim com estas ferramentas torna-se um processo simples, pois utiliza uma sintaxe compreensível de comandos estruturados por meio de uma programação modular, onde os blocos de código são agrupados de acordo com a lógica da ação desejada pelo usuário. Por fim, o programa resultante pode ser carregado ao mundo virtual oferecendo um maior nível de interação entre o usuário e o ambiente imersivo.

\section{Conclusões}


Neste ponto da pesquisa, conclui-se que a utilização de metaversos permite ao usuário um nível de imersão cada vez mais realístico em função da qualidade de seus elementos gráficos, capacidade de renderização e propriedades de integração de diferentes tecnologias.

As peculiaridades observadas nas diferentes fases do estudo possibilitam afirmar que:

- Em relação à instalação e construção dos mundos virtuais, o OpenSim apresentase relativamente estável, possibilitando um uso efetivo e amplo, pois não exige maiores conhecimentos para sua implementação;

- Sobre a importação de objetos 3D, o OpenSim aceita diferentes padrões, porém arquivos .xml são carregados de forma simples e sem erros.

- Um problema claro identificado está nos diferentes visualizadores que podem ser utilizados, visto o fato de que cada um possui peculiaridades e funcionalidades específicas, não aceitas pelos demais. Esta falta de padronização acarreta severas inconsistências nos mundos virtuais criados;

- A programação dos objetos, utilizando as linguagens de script, com programas como o Scratch for OpenSim e ScripTastic permitiram a inserção de altos níveis de interatividade aos elementos, embora os códigos implementados não tenham sido dotados de alta complexidade ou lógica.

\section{Referências}

AMARAL, Erico; AVILA, Barbara; ZEDNIK, Herik; TAROUCO, Liane. Laboratório Virtual de Aprendizagem: Uma Proposta Taxonômica. Renote. v. 10 n. 2, p. 1-10, 2011. BLOOM, Benjamin S. The 2 Sigma problem: The Research for Methods of Group Instruction as Effective as One-to-one Tutoring. Educational Researcher. v. 13, n. 6, p. 4-16, 1984.

CALLAGHAN, Michael, J.; HARKIN, Jim; MCGINNITY, Thomas M.; MAGUIRE, Lion P. Client-se rver architecture for collaborative remote experimentation. Journal of Network and Computer Applications, Northland, v. 30, p. 1295-1308, set/2007. CALLAGHAN, Michael, J.; HARKIN, Jim; MCGINNITY, Thomas M.; MAGUIRE, Lion P. Intelligent User Supportin Autonomous Remote Experimentation Environments. 2008. Trabalho apresentado em IEE Transation Industrial Eletronics, 2008.

Dede, Chris. EcoMUVE. Advancing Ecosystems Science Education via Situated Collaborative Learning in Multi-User Virtual Environments. Disponível em: http://ecomuve.gse.harvard.edu/. Acesso em: 16 de ago, 2012.

Greenbush Labs, Projeto ScripTastic, disponível em: http://scriptastic.greenbush.us/. Acessado em 01 de setembro de 2012.

FLETCHER, J. D. Technology, the Columbus Effect, and the Third Revolution in Learning. IDA Document. Institute for defense analyses. p. 32, 2001.

JONASSEN, D. et alli. Learning with Technology: A Constructivist Perspective. New Jersey: Prentice Hall, 1999.

KESKITALO, Tuulikki; PYYKKÖ, Elli; RUOKAMO, Heli. Exploring the Meaningful Learning of Students in Second Life. In: Educational Technology \& Society, 2011, v. 14. p. 16-26.

KOLB, David A.; BOYATZIS, Richard E.; MAINEMELIS, Charalampos. Experiential Learning Theory:Previous Research and New Directions. In: 1986 
LEAL, David (2007). Mundos Virtuais On-line: Um Mini-guia. Disponível em http://www.masternewmedia.org/pt/2007/04/10/mundos virtuais online um minigui a. htm. Acessado 12/12/2011.

MORGADO, Leonel Caseiro; BARBOSA, Luis; SOUZA, Antonio. Ensaio de utilização do mundo virtual Second Life no ensino de programação de computadores. UTAD Universidade de Trás-os-Montes e Alto Douro, Portugal, 2011.

OPENSIMULATOR. Disponível em: http://opensimulator.org/wiki/Main_Page.

Acesso em: 15 set 2012.

RAMOS-PAJA, Andrés C.; SCARPETTA, José M. R.; MARTÍNEZ-SALAMERO, Luis. Integrated Learning Platformfor Internet-Based Control-EngineeringEducation. 2010. Trabalho apresentado em IEE Transation Industrial Eletronics, 2010.

Schaf, Frederico. M.; Paladini, Suenoni. ; Pereira, Carlos. E. 3D AutoSysLab Prototype A Social, Immersive and Mixed Reality Approach for Collaborative Learning Environments. In: Proceedings of the IEEE International Conference EDUCON, 2012, Marrakesh, Morocco, v. 1. p. 1161-1169.

SCHMITT, Marcelo A. R.; TAROUCO, Liane M. R. Metaversos e laboratórios virtuais - possibilidades e dificuldades. Revista de Novas Tecnologias na Educação, Porto Alegre, v. 6, n. 1, p 1-12, jul/2008.

SIEVERS JUNIOR, Fretz; RAMOS, Eliane S.; GERMANO, José S. E.; OLIVEIRA, José M. P.; PANCCIONI, Bruno; MORZELLI, Nizi V. Simulação do Ambiente WebLab Um laboratório de acesso remoto educacional através de Redes de Petri Coloridas. In: Simpósio Brasileiro de Informática na Educação. n 22, 2011, Aracajú. Anais, Aracajú, 2011. p. 506-515.

VYGOTSKY, Lev S. A Formação Social da Mente. São Paulo: Martins Fontes, 2008. WIJEKUMAR, Kausalai K; Jonassen, David H. The role of computer tools in experts' solving ill-structured problems. Computers in Human Behavior. v. 23, p. 664-704, 2004. 